\title{
TWO CASES OF MONILIAL SEPTICAEMIA, SECONDARY TO CARCINOMA OF THE MOUTH, TREATED SUCCESSFULLY WITH INTRAVENOUS AMPHOTERICIN B
}

\author{
Klaus Lewin, M.B., B.S. \\ Registrar to the Department of Morbid Anatomy, Westminster Hospital
}

\begin{abstract}
MoNILIAL SEPTICAEMIA is becoming a fairly familiar complication of antibiotic, antineoplastic and steroid therapy, although its diagnosis during life has only recently been reported in this country by Dupré, Jones and Penman. It not uncommonly occurs in patients receiving intravenous fluids, in patients who have had an operation and after self-puncture among drug addicts (Boyd and Chappell, r 96r). So far only five instances have been recorded in the literature of a satisfactory response to treatment and therefore we would like to report another two, both of patients with carcinoma of the tongue treated with intra-arterial infusion of vinblastine sulphate.
\end{abstract}

\section{Case I}

A 60-year-old man with a four-year-old growth on the inner aspect of the left gum. In October 1962 the new growth had invaded the tongue and was superficially ulcerated in parts. Metastases were present in the submandibular lymph nodes on both sides. The new growth and glands were treated with a course of external radiation (maximum tumour dose of $3,800 \mathrm{r}$ and minimum dose of 3,100 ) over four weeks at $250 \mathrm{kV}$.

In April 1962 he was admitted to Westminster Hospital for bilateral external carotid catheterization and infusion of vinblastine sulphate. The carcinoma had reappeared and had invaded the floor of the mouth and base of the tongue. Metastases in the submandicular lymph nodes on both sides had also reappeared. At this time a throat swab showed scanty epithelial cells and yielded Escherichia coli but no monilia on culture. The latter was, however, isolated from a rectal swab. His white cell count was 5,200/cu. mm. (neutrophils $82 \%$, lymphocytes 10\%).

Treatment was stopped after five days because of a severe reaction to vinblastine sulphate; and at the same time he developed a pyrexia which did not respond to a week's course of erythromycin and novobiocin. On the tenth day white cell count was 1,300 and Candida albicans was isolated from a blood culture (glucose broth after 72 hours' incubation at $37^{\circ} \mathrm{C}$.). The same species of Candida was also grown from both intraarterial canulæ and a rectal swab.

The patient was given 500,000 units of mycostatin daily for two weeks and $5 \mathrm{mg}$. of intravenous amphotericin $B$. The latter produced severe hypotension, rigors and sweating, and his blood pressure would not rise over $90 \mathrm{~mm}$. $\mathrm{Hg}$ in spite of measures such as raising the foot of the bed and the injection of metaraminol hydrogen tartrate ('Aramine'). The dose of amphotericin $B$ was reduced to $0.5 \mathrm{mg}$. on the following day and thereafter doubled daily. Treatment was stopped after seven days, by which time the pyrexia had settled and blood cultures had become sterile. The total dose of amphotericin B given was $36 \mathrm{mg}$. and the only serious side-effects observed (apart from the hypotension already mentioned) were a marked lethargy and drowsiness experienced by the patient. Both symptoms, however, disappeared within 48 hours of discontinuing the drug.

\section{Case 2}

A 68-year-old man who presented in August 196r with pain on the right side of his tongue. Clinical examination revealed a mass on the floor of the mouth invading the tongue from the right side, but with no lymph node metastases. Three weeks later a lump, presumably a metastasis, had developed in a node of the right carotid triangle. He was treated with megavoltage therapy by two parallel opposed fields centred over the angle of the jaw and received a central dose of 1,000 r in 47 days. By the end of November 1961 the growth in the mouth had virtually disappeared, leaving only a little scarring in the mouth and some tethering of the right side of the tongue.

In April I 962 he was admitted to Westminster Hospital because of dysphagia and intense pain in his right ear and floor of the mouth on the right side. The growth had clearly recurred and invaded the posterior two-thirds of the tongue and the right pharyngeal wall; metastases were obvious in the submandibular lumph nodes on both sides.

The neoplastic area was perfused with vinblastine sulphate via the right external carotid artery for 13 days when the catheter was inadvertently pulled out. On the fifth day of treatment a throat swab grew Candida albicans, $E$. coli and various streptococci. At this time the white cell count was 8,100 cu. mm. with $78 \%$ neutrophils.

At the termination of perfusion the patient was pyrexial and a glucose broth blood culture grew Candida albicans after 72 hours' incubation. This organism was also isolated from his throat swab but not from the intra-arterial catheters. His total white cell count at this time had fallen to $4,500 / \mathrm{cu}$. $\mathrm{mm}$. The patient was now given mycostatin orally (500,000 units six-hourly) and amphotericin $B$ intravenously ( $1 \mathrm{mg}$. on the first day, two on the second, four on the third and thereafter $8 \mathrm{mg}$. for three days). After three days' treatment the patient was afebrile and blood cultures remained sterile, and on the sixth day the drugs were stopped. There were no side-effects of the amphotericin B apart from drowsiness and lethargy.

\section{Discussion}

So far only five instances of Candida albicans fungicæmia have been recorded who have responded favourably to treatment, as this condition is nearly always fatal.

Schaberg, Hildes and Wilt (1955) described a patient suffering from bulbar poliomyelitis with a secondary fungicæmia who remained well after treatment with cycloheximide (Acti-Dione) for two weeks. Louria and Dinee (1960) saw two instances, 
both in patients receiving large doses of antimicrobials and steroids after abdominal operations. In one the organism had almost certainly entered the blood via an intravenous catheter. Both responded satisfactorily to a large dose of intravenous amphotericin $(\mathrm{I}, 500 \mathrm{mg}$.).

Utz and Treger (1959) treated one person with an underlying pyelonephritis with I IO mg. of amphotericin.

Harrell and Thompson (1958) quote one patient cured by mycostatin given intramuscularly.

Braude and Rock (1959), on the basis of four cases, have stated that acute disseminated moniliasis in adults is a definite clinical entity characterized by two outstanding features:

(I) A history of continuous antibiotic treatment during the course of a protracted debilitating illness.

(2) Rapid deterioration of the patient's general condition with the development of fever, shock, gastro-intestinal bleeding and 'depressed sensorium'.

In our two patients the second feature was not present. Although both had a pyrexia which did not respond to antibacterial therapy, they did not deteriorate rapidly, they were not shocked and there was no evidence of gastro-intestinal hæmorrhage. It is possible that we did not encounter these features because the condition was diagnosed soon after its onset.

The increased susceptibility to monilial infection is probably due to alteration of the bacterial flora of the bowel by antibodies and 'virulence enhancement due to corticosteroid therapy' (Bendell and Race, 1961). In our two cases Candida was isolated from the gastro-intestinal tract, suggesting that multiplication on the surface, especially in the mouth, had preceded the infection of the blood. Isolation of this organism from the intra-arterial catheters in the first patient was probably secondary to the fungicæmia, because the wound swab (from the catheter side) was sterile. An interesting feature was the drop in total white cell count in both patients. It seems unlikely that this was caused by the vinblastine sulphate in both cases, since it occurred several days after the drug was discon- tinued and disappeared soon after treatment with amphotericin was started.

In reviewing the treatment of monilia fungicæmia, Utz and Treger (1959) advocate the use of intravenous amphotericin because it is the most powerful anti-monilial drug available and its toxic effects (fever, chills, nausea, rise in blood urea) disappear rapidly after the drug is stopped. Amphotericin is derived from an as yet unidentified species of Streptomyces and probably acts like penicillin (Persellin, Haring and Lewis, 196r; McNall, Halde, Newcomer and Sternberg, 1957-58) by inhibiting cell wall synthesis. Mycostatin is effective locally, but is not absorbed from the gastro-intestinal tract and is very nephrotoxic when given intravenously.

Both of our patients were given mycostatin orally and amphotericin intravenously. The total dose of the latter was under $50 \mathrm{mg}$. in each case, in contrast to the $1,000 \mathrm{mg}$. usually advocated. Side-effects on this dosage were marked lethargy and drowsiness in both patients and an episode of hypotension, rigors and sweating in one.

So many patients are nowadays given steroids, broad - spectrum antibiotics and antineoplastic agents, either alone or in combination, that many instances of monilial septicæmia may well go undiagnosed and, in fact, precipitate death. It would, therefore, seem well to consider this condition in critically ill patients on the above-mentioned drugs as early diagnosis and prompt treatment may wel lead to recovery.

\section{Summary}

Two cases of monilial septicæmia responding to amphotericin B treatment are described: both followed treatment for carcinoma of the tongue with intra-arterial perfusion of vinblastine sulphate and, in one case, broad-spectrum antibiotics.

I am indebted to Professor B. W. Lacey for his constructive criticism and to Mr. G. Westbury and Dr. K. A. Newton of Westminster Hospital for permission to publish the cases under their care.

\section{REFERENCES}

Bendell, W. R., and Race, J. G. (1961): Acute Disseminated Candidiasis in Aplastic Anæmia, Arch. intern. Med., 108, 916.

Boyd, J. F., and Chappell, A. C. (1961): Fatal Mycetosis due to Candida Albicans after Combined Steroid and Antibiotic Therapy, Lancet, i, 19.

Braude, A. I., and Roch, J. A. (1959): Syndrome of Acute Disseminated Moniliasis in Adults, Arch. intern. Med., 104, 107.

Dupre, J., Vaughan Jones, R., and Penman, H. G. (1962): Candida Albicans Septicæmia, Postgrad. med. Y., 38, 176.

HARREL, E. R., and Thompson, G. R. (1958): Systemic Candidiasis (Moniliasis) Complicating Treatment of Bacterial Endocarditis, with Review of Literature and Report of Apparent Cure of One Case with Parenteral Mycostatin, Ann. intern. Med., 49, 207.

Louria, D. B., and DineE, P. (1960): Amphotericin B in the Treatment of Disseminated Moniliasis, Y. Amer. med. Ass., 174, 273.

McNal, E. C., Halde, C., Newcomer, V. D., and Sternberg, J. H. (1957-1958): The Current Status of Amphotericin B in the Treatment of Systemic Fungus Infections. Antibiotics Annual, edited by H. Welch and F. MariIbarney.

Persellin, R. H., Haring, O. M., and Lewis, J. F. (1961): Fungal Endocarditis following Cardiac Surgery, Ann. intern. Med., 54, 127.

Shaberg, A., HiLdes, J. A., and Wilt, J. C. (1955): Disseminated Candidiasis, Arch. intern. Med., 95, 112.

Utz, J. P., and Treger, A. (1959): Current Status of Chemotherapy of Systemic Fungal Disease, Ann. intern. Med., 5I, 1220. 